LAWRENCE LIVERMORE NAT IO N A L LABORATORY

\section{Pulsed X-Ray Exposures and Modeling for Tungsten as an IFE First Wall Material}

J. F. Latkowski, R. P. Abbott, R. C. Schmitt

September 23, 2004

16th Topical Meeting on the Technology of Fusion Energy Madison, WI, United States September 14, 2004 through September 16, 2004 
This document was prepared as an account of work sponsored by an agency of the United States Government. Neither the United States Government nor the University of California nor any of their employees, makes any warranty, express or implied, or assumes any legal liability or responsibility for the accuracy, completeness, or usefulness of any information, apparatus, product, or process disclosed, or represents that its use would not infringe privately owned rights. Reference herein to any specific commercial product, process, or service by trade name, trademark, manufacturer, or otherwise, does not necessarily constitute or imply its endorsement, recommendation, or favoring by the United States Government or the University of California. The views and opinions of authors expressed herein do not necessarily state or reflect those of the United States Government or the University of California, and shall not be used for advertising or product endorsement purposes. 


\title{
PULSED X-RAY EXPOSURES AND MODELING FOR TUNGSTEN AS AN IFE FIRST WALL MATERIAL
}

\author{
J. F. Latkowski ${ }^{1}$, R. P. Abbott ${ }^{1,2}$, and R. C. Schmitt ${ }^{1,2}$ \\ ${ }^{1}$ Lawrence Livermore National Laboratory Livermore, CA, latkowski@llnl.gov \\ ${ }^{2}$ University of California at Berkeley, Berkeley, $C A$
}

Dry-wall inertial fusion energy (IFE) power plants must survive repeated exposure to target threats that include $x$-rays, ions, and neutrons. While this exposure may lead to sputtering, exfoliation, transmutation, and swelling, more basic effects are thermomechanical in nature. In the present work, we use the newly developed RadHeat code to predict time-temperature profiles in a tungsten armor, which has been proposed for use in an IFE power plant. The XAPPER x-ray damage experiment is used to simulate thermal effects by operating at fluences that produce similar peak temperatures, temperature gradients, or thermomechanical stresses. Soft x-ray fluences in excess of $1 \mathrm{~J} / \mathrm{cm}^{2}$ are possible. Using RadHeat, we determine the XAPPER x-ray fluence needed to simulate thermomechanical effects expected in a typical IFE case of interest. Here, we report our findings and detail directions for future experiments and modeling.

\section{INTRODUCTION}

Designers are able to develop chamber wall designs that avert single-shot damage such as melting and ablation, however, this is insufficient for a successful chamber design. Multiple-shot issues such as surface roughening and thermomechanical fatigue are missed in such analyses. In addition, single-shot modeling and experiments easily can miss very small levels of ablation, which would be unacceptable in an operating power plant. For a repetition rate of $5 \mathrm{~Hz}$, the loss of even $0.1 \mathrm{~nm}$ per shot would result in the loss of more than a centimeter per year. In addition, previous work by Zaghloul, Tillack and Mau shows that the laser-induced damage fluence drops from a single-shot limit of $\sim 140 \mathrm{~J} / \mathrm{cm}^{2}$ to $<20 \mathrm{~J} / \mathrm{cm}^{2}$ after $10^{5}$ shots. ${ }^{1}$ A damage limit of $\sim 8 \mathrm{~J} / \mathrm{cm}^{2}$ is projected for $10^{8}$ shots, which is equivalent to 1 year of operation at $3.2 \mathrm{~Hz}$. It seems reasonable to assume that similar effects would occur for first wall materials, and thus, multi-shot studies are required.

For the present work, we use a pulsed x-ray source for material exposures in conjunction with modeling accomplished with the newly developed RadHeat code.

\section{EXPERIMENTAL SET UP}

The source for the XAPPER x-ray experiment was developed and manufactured by PLEX LLC of Cambridge, MA. $^{2}$ When operated with xenon gas, the PLEX source is capable of producing fluences of several $\mathrm{J} / \mathrm{cm}^{2}$ at repetition rates of up to $10 \mathrm{~Hz}$. Operation for millions of pulses at a time is possible. Figure 1 shows the source layout, along with a schematic of the layout within the target chamber. X-rays are generated via a plasma pinch located at the bottom of the main vacuum chamber. A fraction of those $\mathrm{x}$-rays pass through a foil comb and intersect an ellipsoidal condensing optic. The x-rays are subsequently brought to focus upon the sample. The xenon x-ray spectrum extends roughly from $80-140 \mathrm{eV}$, which is significantly softer than the IFE spectrum. It is, however, possible to replicate the peak surface temperatures or thermomechanical stresses expected in an operating IFE power plant. If desired, XAPPER can be operated with either argon or nitrogen plasmas for production of 250-300 or 400-450 eV x-rays, respectively. Either results in a significant reduction in the $\mathrm{x}$-ray fluence at focus.

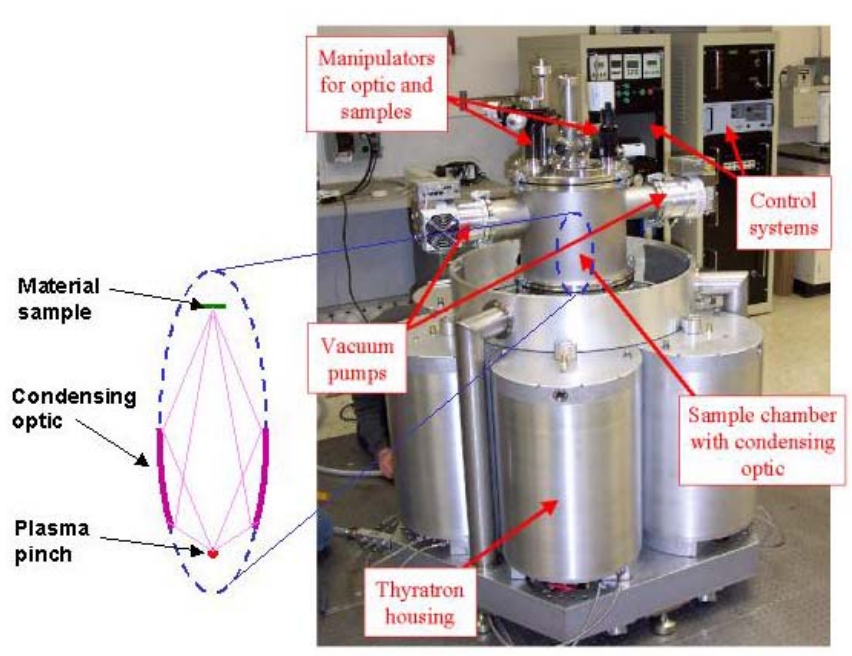

Fig. 1. XAPPER is based upon an ellipsoidal geometry. 
XAPPER is equipped with a 1-m grazing-incidence $\mathrm{x}$-ray spectrometer from McPherson, a Scientech vacuum calorimeter, and a back-thinned vacuum-compatible charge-coupled device (CCD) camera from Princeton Instruments/Roper. Using the spectrometer, we have demonstrated tuning of the $\mathrm{x}$-ray fluence and spectrum by changing the discharge voltage and/or gas injection pressure. $^{3}$ Additionally, it is possible to filter the unfocused beam or intentionally misalign the condensing optic to obtain a variety of x-ray fluences. Calorimeter results show a per-pulse x-ray energy of $25 \mathrm{~mJ}$. CCD camera images, such as that shown in Figure 2, show spot sizes of $\sim 1 \mathrm{~mm}$ for the optic in the reversed (demagnifying) configuration and $\sim 2.5 \mathrm{~mm}$ with the optic in the original (magnifying) configuration. This leads to measured fluences in the range of $0.7-3.8 \mathrm{~J} / \mathrm{cm}^{2}$. Onedimensional calculations suggest that a fluence of only $\sim 1$ $\mathrm{J} / \mathrm{cm}^{2}$ is required to melt tungsten in a single shot.

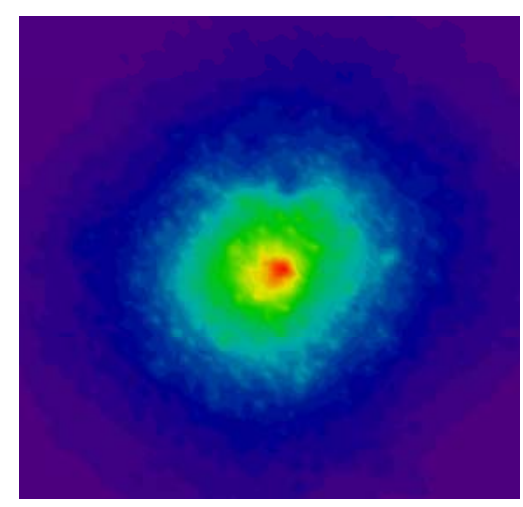

Fig. 2. CCD images show x-ray spot sizes of 1-2.5 mm.

The XAPPER chamber was recently upgraded to provide approximately four times the volume under vacuum. This upgrade enables us to field the CCD camera, calorimeter and up to four samples (one can be heated) at a time. Thus, we are now able to make a fluence measurement and subsequently expose samples without coming up to atmosphere and potentially altering the optical alignment or focus.

Soon, XAPPER will be provided with a non-contact optical thermometer, which has been developed and constructed by the University of California at San Diego. The thermometer provides a temporal resolution of $\sim 1 \mathrm{ns,}$ and thus, it will enable us to resolve the temporal history of irradiated samples.

Our ability to precisely align the ellipsoidal condensing optic has improved to the point at which we are capable of producing single-shot melting on XAPPER. Figure 3 shows a tungsten sample $(5.7 \times 6.2$ $\mathrm{mm}$ in size) that was exposed, at different locations, to a varying number of shots ranging from 1 to 30,000 .

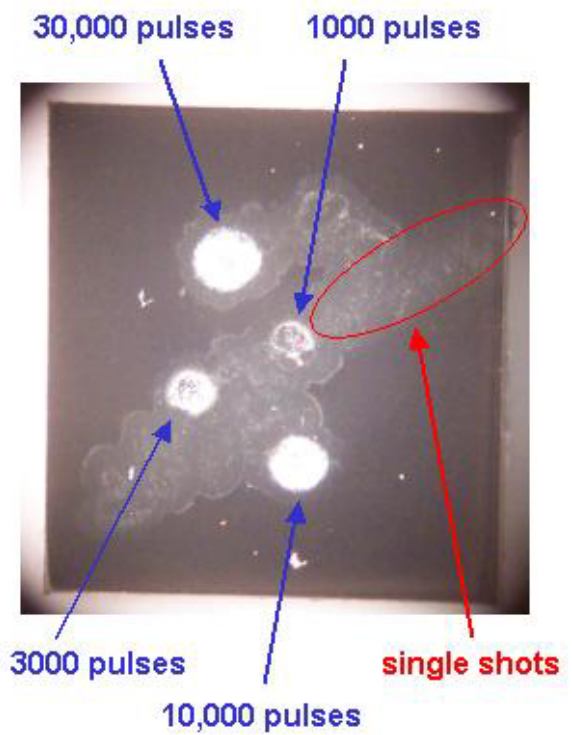

Fig. 3. With improved optical alignment, XAPPER is capable of melting of tungsten in a single shot.

\section{COMPUTATIONAL MODELING}

In order to direct experiments, it is essential to have predictions of the temperature and stress state temporal histories expected to result from an actual IFE target. The reference target output calculations have been completed by L. J. Perkins at Lawrence Livermore National Laboratory. ${ }^{4,5}$ The target output consists of neutrons, ions and x-rays. Due to scattering within the target, the neutron output falls from $80 \%$ to $69 \%$. X-ray output is approximately $1.4 \%$ of the target yield, and the ion output makes up the balance. The ions consist of burn (fast) and debris (slow) ions. Species include protons, deuterons, tritons, alphas, ${ }^{3} \mathrm{He}$, carbon and gold or platinum for high$\mathrm{Z}$ coated targets. To accurately determine the temperature and stress histories in an IFE first wall, it is necessary to account for each of these species, along with their stopping through the chamber background gas, if any. We have developed the RadHeat code to rapidly model such systems, including multiple pulses.

\section{III.A. RadHeat Overview}

Radheat is a finite difference heat transfer code that can determine the transient temperature evolution of multi-material targets in pulsed penetrating radiation environments. It makes use of energy-dependent opacity data and detailed stopping tables to accurately model the volumetric deposition of any number of photon or ion spectra. The heat diffusion equation is solved implicitly and timestep-dependent stability issues are, therefore, eliminated. This allows simulations to achieve high fidelity during times of thermal activity and efficient 
speed elsewhere. Convective and radiative cooling of target surfaces is handled as well as the ability to impose any initial temperature profile. The essential physical model for which RadHeat is applicable is illustrated in Figure 4. RadHeat is able to handle an arbitrary number of layers, each of which is sub-divided into multiple zones. Boundary conditions at the front and back surfaces are specified, as well as pulsing repetition rates and the total number of pulses to be analyzed.

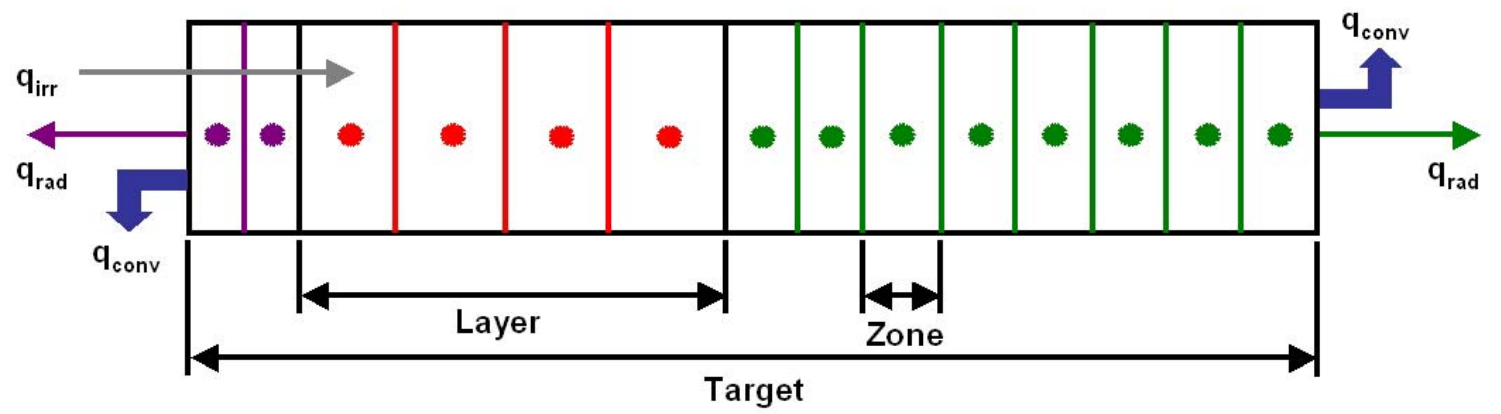

Fig. 4. A RadHeat analysis includes any number of layers, which may be sub-divided into multiple zones.

RadHeat uses temperature-dependent properties found on the ARIES web site. ${ }^{6}$ Ion stopping powers are taken from tables generated using the SRIM code of J. F. Ziegler et al. $^{7}$

In future work, the temperature profiles provided by RadHeat could be coupled to supplementary physics modules to determine stress states, fatigue lifetimes, and even elastic wave propagation in highly transient regimes. RadHeat has been benchmarked against a suite of analytical solutions, and the first round of validation experiments will be completed once the optical thermometer has been installed.

\section{III.B. RadHeat Results}

The base case IFE first wall consists of a tungsten armor that is $1-\mathrm{mm}$-thick mounted to $3.5 \mathrm{~mm}$ of ferritic steel. Issues of interest include the peak surface temperature and stress, as well as the temperature rise at the tungsten/steel interface. A suite of calculations have been completed for various conditions, but results are shown for the following case: $154 \mathrm{MJ}$ target yield, $8 \mathrm{~m}$ radius target chamber, and $1.3 \mathrm{~Pa}$ (10 mTorr) of xenon background gas within the chamber. A repetition rate of $10 \mathrm{~Hz}$ is assumed. The surface zone is defined as the first $10 \mathrm{~nm}$ of tungsten.

Figure 5 shows the temperature history for several pulses. Note that, due to the scale, one can see the details of the first pulse, but only the peak can be seen for the next nine pulses. The surface temperature rises by only a small amount at $\sim 27 \mathrm{~ns}$ due to the arrival of the prompt $\mathrm{x}$ rays. At about $1 \mu \mathrm{s}$, however, the temperature begins its rapid rise to approximately $3000 \mathrm{~K}$. The temperature falls nearly back down to its starting point by the time of the next pulse, but note that each pulse reaches a slightly higher peak. This "ratcheting" effect is due to the system not yet reaching a balance between the incoming power and the cooling at the back of the tungsten/steel armor. Equilibrium is reached after several hundred pulses. The discrete steps seen within the first pulse represent the arrival of different ion species, each with their own timeof-flight.

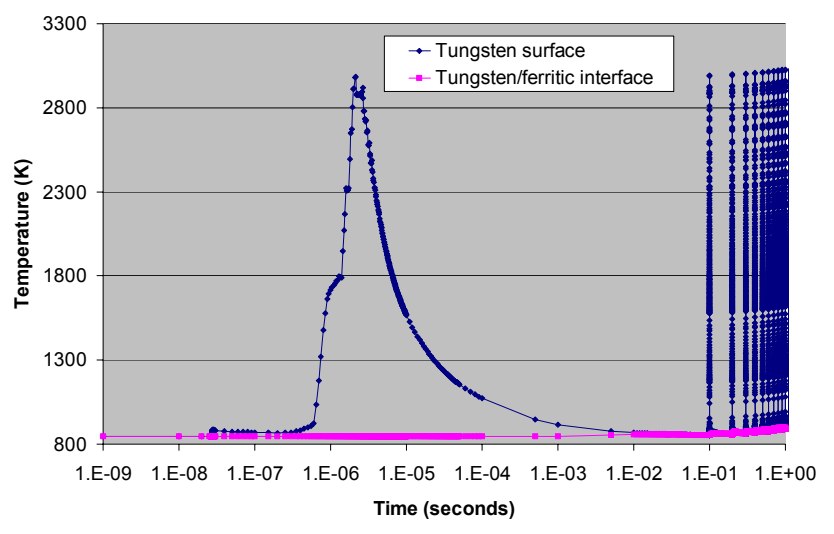

Fig. 5. X-rays have relatively little effect at $\sim 27 \mathrm{ns,} \mathrm{but}$ the arrival of debris and burn ions raises the tungsten surface temperature to $\sim 3000 \mathrm{~K}$.

If one zooms in on the temperature axis and switches the time axis to a linear scale, it is possible to see the temperature rise at the tungsten/steel interface. As shown in Figure 6, a temperature rise of $\sim 15 \mathrm{~K}$ is expected for each pulse. The interface eventually ratchets up to a steady-state temperature of $960 \mathrm{~K}$, assuming a back surface (blanket) heat transfer coefficient of $10 \mathrm{~kW} / \mathrm{m}^{2}-\mathrm{K}$. Due to differential expansion, stress occurs at the interface, which may act towards debonding of the armor. Fortunately, experiments suggest that the bonding can be robust against such stresses. ${ }^{8}$ 


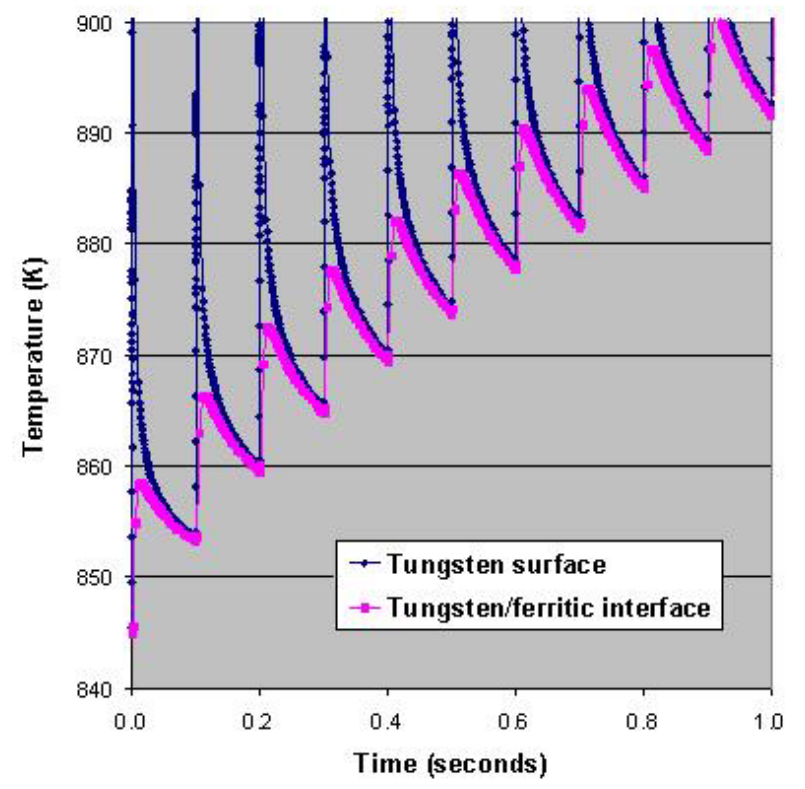

Fig. 6. A per-pulse temperature rise of $\sim 15 \mathrm{~K}$ is expected at the tungsten/ferritic steel interface.

By adjusting the fluence on XAPPER, one can match the peak temperatures expected for the tungsten surface in an IFE power plant. Specifically, an x-ray fluence of $0.7 \mathrm{~J} / \mathrm{cm}^{2}$ comes close to matching the peak temperature of nearly $3000 \mathrm{~K}$. Figure 7 shows a RadHeat results for $10 \mathrm{~Hz}$ XAPPER exposures at this fluence.

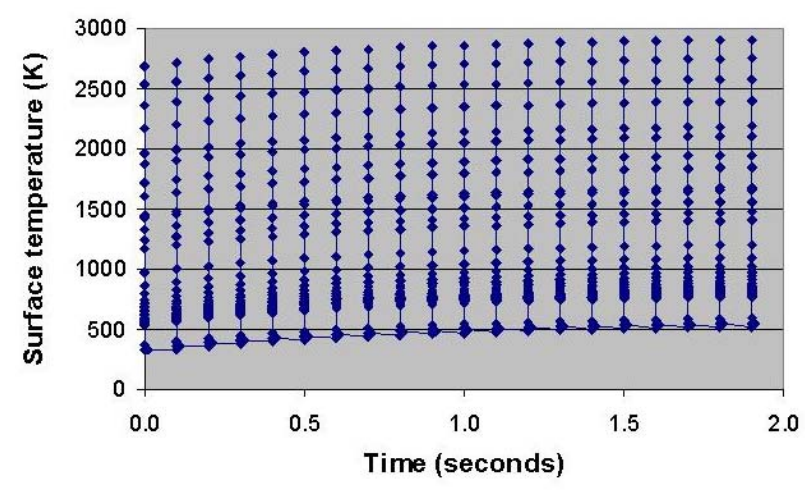

Fig. 7. XAPPER can be operated at $\sim 0.7 \mathrm{~J} / \mathrm{cm}^{2}$ and $10 \mathrm{~Hz}$ to match the expected peak surface temperature.

\section{EXPERIMENTAL RESULTS}

A campaign of $\mathrm{x}$-ray exposures was carried out using XAPPER. Both single crystal and powder metallurgical tungsten samples were exposed to a varying number of pulses at a fluence of approximately $0.7 \mathrm{~J} / \mathrm{cm}^{2}$. The pulseto-pulse jitter was observed to be $<15 \%\left(\sim 0.1 \mathrm{~J} / \mathrm{cm}^{2}\right)$, but this will have to be better quantified in future experiments. Note that the samples were not actively heated other than by the x-ray beam. As a result, the temperature falls back down close to room temperature after each shot. Actively heated samples will be used in future experiments.

Exposed and unexposed portions of each sample were examined using white-light interferometry. Surface roughness measurements were made and are reported here. Prior to irradiation, the single crystal samples were extremely smooth with a roughness of $\sim 10 \mathrm{~nm}$ rms. The powder metallurgical samples were twice as rough at $20 \mathrm{~nm}$ rms. Figure 8 shows how the surface roughness increased with the number of pulses.

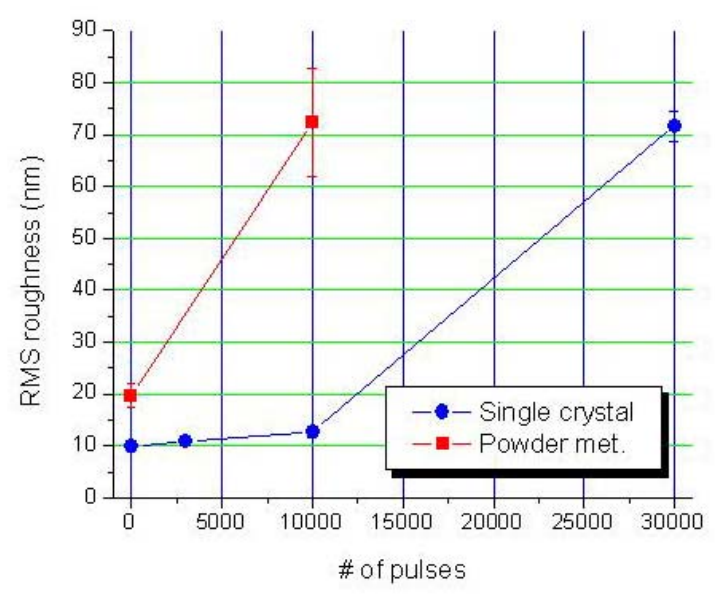

Fig. 8. The surface roughness grows with an increasing number of $\mathrm{x}$-ray pulses.

\section{SYNTHESIS AND FUTURE WORK}

The results shown in Figure 8 raise a number of interesting issues and questions. First, it is interesting to note that the use of single crystal material seems to provide some sort of threshold effect. That is, the material does not appear to roughen until a certain number of pulses $(>10,000$ in these experiments) have been experienced. Second, the single crystal sample roughens to the same degree $(\sim 70 \mathrm{~nm} \mathrm{rms})$ as the powder metallurgical sample. While one can speculate that the powder metallurgical sample roughens by means of slipping grain boundaries, it would be interesting to know the mechanism of roughening for the single crystal sample.

Other interesting questions are raised by these results:

- Will the roughening continue linearly, will the rate of roughening increase, or will it eventually saturate?

- Will the single crystal sample continue to roughen more slowly than the powder metallurgical sample?

- How does the roughening compare at lower and higher x-ray fluences? 
- Will the roughening be the same for heated samples?

- What is the mechanism for roughening?

- Is material actually being lost or simply being rearranged?

Future experiments will attempt to answer many of these questions. Specifically, experiments will be completed for powder metallurgical and single crystal tungsten at several different fluences for exposures ranging from 100 pulses up to as many as 300,000 pulses. These experiments will include the use of witness plates, which will be tested for material that might be lost from the samples. Eventually, samples will be actively heated to avoid jumping through tungsten's ductile-to-brittle transition temperature on each and every pulse.

Future experiments also will include use of the noncontact optical thermometer for in-situ measurements of the tungsten surface temperature.

RadHeat development will continue with addition of a stress state module. This will allow us to select XAPPER x-ray fluences that will match the expected IFE stress state rather than simply the peak surface temperature. Assuming that thermomechanical fatigue is a key consideration, then the stress state is the appropriate figure of merit. RadHeat benchmarking and validation will continue as described above.

\section{ACKNOWLEDGMENTS}

The authors would like to thank Dr. Lance Snead of Oak Ridge National Laboratory for providing high-quality tungsten samples for the experiments described herein. We would also like to thank Dr. James Dunn for lending us the CCD camera that was used in the spot size measurements for this work. Finally, Dr. Stephen Payne is thanked for his valuable input and assistance in getting
XAPPER to its current state. Work performed under the auspices of the U. S. Department of Energy by University of California Lawrence Livermore National Laboratory under Contract W-7405-Eng-48.

\section{REFERENCES}

[1] M. McGEOCH, "Radio-Frequency-Preionized Xenon Z-Pinch Source for Extreme Ultraviolet Lithography," Appl. Optics 37, 1651 (1998).

[2] M. R. ZAGHLOUL, M. S. TILLACK, and T. K. MAU, "Laser-Induced Damage of Metal Mirrors Under Long-Term Exposure at Shallow Angle of Incidence," 19th SOFE, Atlantic City, NJ, Jan. 21-25, 2002.

[3] J. F. LATKOWSKI, R. P. ABBOTT, S. A. PAYNE, S. REYES, R. C. SCHMITT, and J. A. SPETH, "Rep-Rated X-Ray Damage and Ablation Experiments for IFE and ICF Applications," Inertial Fusion Sciences and Applications, Monterey, CA (2003).

[4] L. J. Perkins, personal communication.

[5] Target spectra for reference targets can be found at http://aries.ucsd.edu/ARIES/DOCS/ARIESIFE/SPECTRA

[6] Temperature-dependent properties from the ARIES web site have been used: http://wwwferp.ucsd.edu/LIB/PROPS/

[7] J. F. ZIEGLER, J. P. BIERSACK and U. LITTMARK, The Stopping and Range of Ions in Solids, Pergamon Press, New York (1985).

[8] L. SNEAD, G. R. ROMANOSKI, C. A. BLUE, and J. BLANCHARD, "IFE First Wall Survival: Development and Testing of a Refractory Armored Ferritic," presented at the American Nuclear Society's 16th Topical Meeting on the Technology of Fusion Energy, Madison, WI (2004). 